\title{
Ultra-deep through-skull mouse brain imaging via the combination of skull optical clearing and three-photon microscopy
}

Mubin $\mathrm{He}^{1 \#}$, Dongyu $\mathrm{Li}^{2 \#}$, Zheng Zheng ${ }^{3 \#}$, Hequn Zhang ${ }^{1,4}$, Tianxiang $\mathrm{Wu}^{1}$, Weihang Geng ${ }^{1}$, Zhengwu $\mathrm{Hu}^{2}$, Zhe Feng ${ }^{1}$, Shiyi Peng ${ }^{1}$, Liang $\mathrm{Zhu}^{4}$, Wang $\mathrm{Xi}^{4}$, Dan $\mathrm{Zhu}^{2}$, Jun Qian $^{1 凶}$

${ }^{1}$ State Key Laboratory of Modern Optical Instrumentations, Centre for Optical and Electromagnetic Research, College of Optical Science and Engineering, International Research Center for Advanced Photonics, Zhejiang University, Hangzhou 310058, China.

${ }^{2}$ Britton Chance Center for Biomedical Photonics, Wuhan National Laboratory for Optoelectronics, MoE Key Laboratory for Biomedical Photonics, Huazhong University of Science and Technology, Wuhan, Hubei 430074, China.

${ }^{3}$ School of Chemistry and Chemical Engineering, Hefei University of Technology, Hefei 230009, China.

${ }^{4}$ Interdisciplinary Institute of Neuroscience and Technology (ZIINT), College of Biomedical Engineering and Instrument Science, Zhejiang University, Hangzhou 310027, China.

\# These authors contributed equally: Mubin He, Dongyu Li, Zheng Zheng.

ఐEmail: qianjun@zju.edu.cn

\begin{abstract}
Optical microscopy has enabled in vivo monitoring of brain structures and functions with high spatial resolution. However, the strong optical scattering in turbid brain tissue and skull impedes the observation of microvasculature and neuronal structures at a large depth. Herein, we proposed a strategy to overcome the influence induced by the high scattering effect of both skull and brain tissue via the combination of skull optical clearing (SOC) technique and thee-photon fluorescence microscopy (3PM). The Visible-NIR-II compatible Skull Optical Clearing Agents (VNSOCA) we applied reduced the skull scattering and water absorption in long wavelength by refractive index matching and $\mathrm{H}_{2} \mathrm{O}$ replacement to $\mathrm{D}_{2} \mathrm{O}$ respectively. 3PM with the excitation in the $1300-\mathrm{nm}$ window reached $1.5 \mathrm{~mm}$ cerebrovascular imaging depth in cranial window. Combining the two advanced technologies together, we achieved so far the largest cerebrovascular imaging depth of $1.0 \mathrm{~mm}$ and neuronal imaging depth of $>700 \mu \mathrm{m}$ through intact mouse skull. Dual-channel through-skull imaging of both
\end{abstract}

\footnotetext{
${ }^{\circledR}$ Corresponding author: Jun Qian, qianjun@zju.edu.cn
} 
bioRxiv preprint doi: https://doi.org/10.1101/2021.12.20.473469; this version posted December 24, 2021. The copyright holder for this preprint (which was not certified by peer review) is the author/funder, who has granted bioRxiv a license to display the preprint in perpetuity. It is made available under aCC-BY-NC-ND 4.0 International license.

brain vessels and neurons was also successfully realized, giving an opportunity of non-invasively monitoring the deep brain structures and functions at single-cell level simultaneously. 


\section{Introduction}

Brain structural and functional imaging is essential for monitoring neuronal circuitry and pathogenesis of brain diseases including Alzheimer's disease and Parkinson's disease ${ }^{1-6}$. Optical imaging is optimal for its high spatial resolution, real-time performance and free of ionizing radiation. However, the strong scattering effect of biological tissues ${ }^{7}$ has impeded it from observing vasculature and neuronal structures at a large depth. Many technologies have been developed to reduce the scattering effect, including photoacoustic tomography ${ }^{8-10}$ with acoustic penetration, optical coherent tomography ${ }^{11-14}$ with optical heterodyne amplification, and so on. However, these modalities performed poorly in neuronal structure imaging due to the lack of specific mark to neuron, let alone neuronal dynamic imaging.

The mouse cortex is mainly protected by the skull, which exhibits strong scattering, causing a severe limitation for large-depth and high-contrast optical imaging. In fact, several technologies have been proposed to avoid the influence of optical scattering caused by the skull, including the open-skull glass window ${ }^{15}$, the thinned-skull cranial window ${ }^{16,17}$ and the skull optical clearing window ${ }^{18,19}$. The open-skull glass window, which is formed by removing a part of skull and replacing with a glass coverslip, tends to induce a series of inflammatory reactions and increase intracranial pressure ${ }^{20}$. The thinned-skull cranial window, which is literally formed by thinning the skull, requires operating repeatedly due to bone growth and is difficult to use ${ }^{16}$. However, the skull optical clearing window, which locally applies some chemical agents to the skull for refractive index matching, is safe and repeatable, without any craniotomy ${ }^{18}$. Previously developed skull optical clearing windows have enabled repeated imaging of the dendritic protrusions, microglia dynamics and blood capillaries, combined with optical microscopy ${ }^{18,19}$. Nevertheless, most of them employed $\mathrm{H}_{2} \mathrm{O}$ as solution, which has large absorption coefficient in near-infrared region, especially beyond $1300 \mathrm{~nm}$. This would inevitably cause adverse impact on optical imaging with near-infrared excitation or emission.

In addition, mouse brain tissue also exhibits strong scattering effect and impedes deep imaging, unable to be resolved by in vivo tissue optical clearing technology at present. Fortunately, three-photon fluorescence microscopy (3PM) is a typical way for deep imaging with high contrast in turbid tissue ${ }^{21}$. The character of long-wavelength excitation light with low tissue scattering guarantees 3PM large penetration depth, thus maintaining well focus efficiency in turbid tissue ${ }^{22}$. Meanwhile, high-order nonlinear optical effect of three-photon fluorescence (3PF) ensures the localized excitation, and therefore improves imaging resolution and contrast. The two acknowledged long-wavelength excitation windows for 3PM are the 1300-nm and 1700-nm spectral ranges ${ }^{23}$, both of which have long attenuation length at brain tissue. Among the two excitation windows, the 1300-nm window is favored for its lower 
tissue absorption induced heating effect. Moreover, considering universal commercial fluorescent probes for neuronal labeling, the 1300-nm window is more commonly used in 3PF neuronal structural and functional imaging ${ }^{24,25}$, and more capable of simultaneous multi-color imaging ${ }^{26}$. However, 3PM using the 1300-nm excitation under the intact skull currently achieved only $\sim 300 \mu \mathrm{m}$ neuronal imaging depth ${ }^{25}$ and $510 \mu \mathrm{m}$ cerebrovascular imaging depth ${ }^{25}$ owing to the low excitation efficiency.

Herein, we combined in vivo skull optical clearing (SOC) technique and 1300-nm excited 3PM to overcome the scattering and absorption of the skull as well as brain during deep-cortical visualizing. Skull scattering reduction could improve the performance of 3PM effectively according to Monte Carlo simulation results. The Visible-NIR-II compatible Skull Optical Clearing Agents (VNSOCA) we applied introduced $\mathrm{D}_{2} \mathrm{O}$ solution to replace $\mathrm{H}_{2} \mathrm{O}$ solution. Therefore, the light scattering and absorption of skull in the 1300-nm window were both reduced effectively. Afterwards, we realized largest 3PF through-skull cerebrovascular imaging depth $(1.0 \mathrm{~mm})$, assisted by a kind of fluorescent probe with large three-photon absorption cross section. Meanwhile, 3PF through-skull neuronal imaging also improved to $>700 \mu \mathrm{m}$ depth. With prominently reduced scattering effect and near-infrared absorption of skull, 3PM in the 1300-nm window achieved dual-channel deep imaging of both brain vessels and neurons under the intact skull for the first time.

\section{Results}

\section{Skull scattering reduction improved performance of through-skull 3PM}

Skull scattering is one of the major barriers that constrains the through-skull imaging quality. And SOC technique could reduce the skull scattering effectively. Herein, we performed Monte Carlo simulation to investigate how SOC would enhance the performance of three-photon excitation due to the better focusing inside brain tissue. Fig. 1a shows the simulated distribution of photons when the 1300-nm light was focused on the brain tissue through skull with the scattering of skull decreasing. Fig. 1b states the situation when a 1700-nm light was focused on the brain tissue. It was obvious that reducing the skull scattering would effectively reduce the out-of-focus light both for 1300-nm window and 1700-nm window. In addition, quantitative analysis demonstrated that, both the photon intensity and photon density at the focus point were getting better as the skull scattering coefficient decreased in the 1300-nm window (Fig. 1c and 1d) and the 1700-nm window (Fig. 1e and 1f). In addition, we simulated the amount of signal intensity left at the skull surface after penetrating through brain tissue and skull when the emission was generated at certain depth of brain. As shown in in Supplementary Figure 1, the signal photons showed similar distribution on the surface as the skull scattering coefficient decreased. However, the overall photons penetrating successfully to the surface obviously 
increased as the skull scattering coefficient decreased (Supplementary Figure 2). Simulation results above verified the skull scattering reduction induced by SOC could promisingly increase the photon density of the three-photon excitation light at focus point, and decrease the attenuation of the generated three-photon emission signals before detected.

Light absorption by water in near-infrared region was another factor that should be considered while applying the skull optical clearing agent (SOCA) to 3PM. Since deuterium oxide $\left(\mathrm{D}_{2} \mathrm{O}\right)$ has been applied as an immersion medium in near-infrared imaging for its reduced absorption in near-infrared region ${ }^{27}$ (Fig. 1g), in this work we prepared visible-NIR-II compatible skull optical clearing agents (VNSOCA) which replaced $\mathrm{H}_{2} \mathrm{O}$ in urea-based skull optical clearing agents (USOCA) with deuteroxide solution. Then we used Monte Carlo simulation to compare two SOCAs to investigate the impact of absorption of the SOCA (Fig. 1h and 1i) on 3PM. As shown in Supplementary Figure 3, after SOC treatment, solution 2 (S2) of the SOC was remained on the skull for imaging. Therefore, simulations were performed to investigate the 1300-nm and 1700-nm light focusing ability when S2 of USOCA and VNSOCA covered skull. As shown in Supplementary Figure 4, compared with USOCA, VNOSCA can increase the 1300-nm photon density at the focal point by 1.25 times. Similarly, as shown in Supplementary Figure 5, VNSOCA can increase the 1700-nm photon density at the focal point by 2 times. However, in the case of VNSOCA, the absolute value of 1700-nm photon density was lower than that of 1300-nm photon density at the focal point $\left(1.4 \times 10^{4}\right.$ VS $\left.3.1 \times 10^{4}\right)$, due to stronger brain tissue absorption.

Furthermore, we also performed ex vivo 3PM test to verify the skull clearing effect by VNSOCA. A kind of lab-synthesized aggregation-induced emission (AIE) nanoparticle (NP), DCBT NP (the details will be discussed later), and a lab-built three-photon microscopic system (supplementary Figure 6), were used for the test. The results indicated that, covered by an intact skull, the 3PF signals of DCBT NPs-filled capillary were rather weak. On the contrary, when the skull was treated by VNSOCA, the signals were remarkably increased (Supplementary Figure 7). In addition, VNSOCA treatment also increased the signal-to-background ratio (SBR, supplementary Figure 8) and the resolving power (supplementary Figure 9) of 3PM.

Overall, VNOSCA assisted skull optical clearing could reduce both light absorption by water in near-infrared region and skull scattering, therefore enhanced the performance of through-skull 3PM.

\section{The advantages of 1300-nm excitation window over 1700-nm excitation window}

The advantages of long excitation wavelength and high-order nonlinear optical 
confinement attributed to 3PM were effective ways to overcome the scattering effect in deep brain imaging. The greatest advantage of the 1300-nm excitation window against the 1700-nm excitation window is its much lower tissue absorption. The Monte Carlo simulation of temperature rising caused by tissue absorption of excitation light was shown in Fig. 2a-b and supplementary Figure 10. Compared with 1700-nm window, temperature rising in the 1300-nm window was obviously milder. Also, the temperature distribution in the 1300-nm window was more dispersive, avoiding local overheating at focused point. On the contrary, 1700-nm light would cause a sharp temperature rise near the focus, where overheated damage tended to happen easier.

Another advantage of the 1300-nm excitation window was that compared with the 1700-nm excitation window, the 1300-nm excitation window should obtain higher excitation efficiency and fluorescence intensity for its shorter wavelength according to the multi-photon fluorescence photon flux formula ${ }^{28}$. However, the low three-photon absorption cross-section $\left(\sigma_{3}\right)$ of most fluorescence probes restricted their 3PF intensity from further strengthening. Fortunately, we currently developed an AIE probe named DCBT with large $\sigma_{3}$ which was realized by the intra- and intermolecular synergistic engineering. The AIE character of DCBT endows it with bright fluorescence and high photostability in the aggregated nanoparticles. Considering those unique properties, DCBT was introduced in this study and its $\sigma_{3}$ in the 1300-nm and 1700-nm excitation windows were evaluated. As shown in supplementary Figure 11, DCBT were encapsulated by F-127, which was approved by the US Food and Drug Administration (FDA), to form amphipathic organic nanoparticles. The results of dynamic light scattering (DLS, $36.2 \pm 1.9 \mathrm{~nm}$, Zetasizer Nano-ZS) and scanning transmission electron microscopy (STEM, $32.3 \pm 3.88 \mathrm{~nm}$, tecnai Spirit) in supplementary Figure 12 showed the micellar systems were uniformly distributed. The extinction and photoluminescence (PL) spectra were displayed in Fig. 2c. The quantum yield of DCBT NPs was measured as $3.17 \%$. Afterwards, we measured its nonlinear emission spectrum. As shown in Fig. 2d, under the 1300-nm fs excitation, DCBT NPs generated red emission with a peak at $\sim 640 \mathrm{~nm}$. In addition, the dependence relationship between the nonlinear fluorescence intensity and the excitation light intensity was measured. As shown in Fig. 2e, the logarithm of the emission intensity against that of the excitation intensity showed a linear slope of approximately 2.95, indicating a major three-photon absorption process under 1300-nm fs excitation. Moreover, 3PF intensity decreased by only 30\% over 16 minutes of continuous irradiation (Fig. 2f), suggesting the promising photostability of DCBT NPs for 3PM.

Furthermore, $\sigma_{3}$ of DCBT in aggregation at various wavelengths were measured, since it was the major feature manifesting the 3PM capability of an optical probe. 
Results showed that, the $\sigma_{3}$ of DCBT at $1300 \mathrm{~nm}$ was much larger than that at 1700 $\mathrm{nm}$ (Fig. 2g). The largest $\sigma_{3}$ at $1300 \mathrm{~nm}$ within the test range was calculated to be 1.26 $\times 10^{-77} \mathrm{~cm}^{6} \mathrm{~s}^{2}$, which is the highest value among the reported organic probes ${ }^{26,29}$. Hence, 3PF intensity excited at the 1300-nm wavelength was $~ 10$ times higher than that excited at the 1700-nm wavelength (Fig. 2h). Therefore, with the help of the improved $\sigma_{3}$ of fluorescence probe, in vivo 3PF imaging in the 1300-nm window would be more suitable for the observation of brain tissue with the high scattering.

\section{In vivo deep 3PF brain vasculature imaging of the mouse with the open-skull glass window}

With the assistance of DCBT NPs and utilization of open-skull glass window (Fig. 3a), we adopted 3PM to perform ultra-deep visualization of mouse cerebral vessels. The mouse was imaged after injected with DCBT NPs dispersion $(1.5 \mathrm{mg} / \mathrm{mL}, 0.2$ $\mathrm{mL}$ ). Fig. 3b showed 3D reconstruction of cerebral vessels under the excitation of 1700-nm window and 1300-nm window. The imaging depth of 3PM using the 1700-nm window only reached $1.1 \mathrm{~mm}$ (Supplementary Figure 13) due to the low three-photon action cross-section. In contrast, 3PM performed with the 1300-nm window could reach $1.5-\mathrm{mm}$ imaging depth. This imaging depth has already passed through the white matter layer and reached the hippocampus regions, and broke the previously reported organic probe-assisted 3PF imaging depth record $\left(1.4 \mathrm{~mm}^{29}\right)$ using the 1300-nm window.

Brain vessels at various depth was shown in Fig. 3c-j. The tiny blood capillary with a size of $3.7 \mu \mathrm{m}$ could be distinguished at the $1.5 \mathrm{~mm}$ depth (Fig. $3 \mathrm{j}$ and Supplementary Figure 14). Images at neocortex layer maintained extremely high SBR due to the background-free feature of $3 \mathrm{PM}$ as shown in Fig. 3k. Some large blood vessels were observed from white matter layer $(800 \mu \mathrm{m}$ in Fig. 3e) to hippocampus layer (1000 $\mu \mathrm{m}$ in Fig. 3f), which was consistent with the results in previous 3PF deep imaging works ${ }^{30-32}$. Because of the higher scattering effect of white matter, SBR appeared to decrease when the region of interest (ROI) travelled through it (Fig. 3k). Such situation is similar to what was observed in long-wavelength excited confocal fluorescence imaging ${ }^{33}$.

Nevertheless, even with relatively low SBR $(\sim 2)$, the vessels were still distinguishable. 3D reconstruction of brain vessels in the 1.1-1.3 and 1.3-1.5 mm was displayed in Fig. 31-m. Large blood vessels extended up to $1.5 \mathrm{~mm}$ below was displayed clearly. However, it was the working distance of the objective $(2 \mathrm{~mm})$ that constrained imaging depth available in our experiment. As demonstrated by the results above, the scattering effect of brain was effectively overcome by 3PM with the improved $\sigma_{3}$ of DCBT NPs in the 1300-nm excitation window. 


\section{In vivo deep brain 3PM under the 1300-nm excitation window with the optical clearing window}

Combining the SOC technique and 3PM together, we performed 3PF microscopic imaging of the mouse with the skull optical clearing window. The schematic of mouse head imaging was displayed in Fig. 4a. The typical bright field view of the mouse brain vasculature after skull clearing in Fig. $4 \mathrm{~b}$ showed more apparent than that before skull clearing. Intensity analysis of vasculature under the VNSOCA treated skull in Fig. 4c demonstrated four distinct brain vessels, which were almost invisible under the untreated turbid skull. Afterwards, the 3PF cerebrovascular imaging before and after skull clearing were carried out on mouse injected with DCBT NPs. As Fig. 4d-g showed, 3PF imaging performed through the original skull had only reached $550 \mu \mathrm{m}$, which was comparable with the previously reported imaging depth ${ }^{25}$. On the contrary, after VNSOCA treatment on the skull to decrease its scattering, 3PF imaging of the same field of view showed enhanced signal intensity and optimized SBR (Fig. 4h-o), especially at large depth. The final 3PF imaging depth reached $1.0 \mathrm{~mm}$ through the skull optical clearing window (Fig. 4p-s). The small blood vessel with a diameter of $4.5 \mu \mathrm{m}$ at $700-\mu \mathrm{m}$ depth was clearly visualized (Fig. $4 \mathrm{p}, \mathrm{t}$ ). Moreover, a 10.4- $\mu \mathrm{m}$ vessel at $1000 \mu \mathrm{m}$ depth could also be distinguished (Fig. 4s, u). 3D reconstruction of brain vessels from 0 to $1000 \mu \mathrm{m}$ was displayed in Fig. 4v. To the best of our knowledge, our 3PM achieved the largest millimeter-level imaging depth under the intact skull, thanks to the reduced skull scattering induced by VNSOCA and reduced brain tissue scattering overcome by $3 \mathrm{PF}$.

Apart from cerebrovascular imaging, visualization of mouse brain neurons at neocortex layers was also conducted under the intact skull. As shown in Fig. 5a-d, not only the signal intensity, but also the SBR and resolution of neuronal dendrite were considerably enhanced at shallow depth after skull optical clearing. Fig. 5e-f showed that the skull optical clearing treatment introduced a 6-time improvement of the 3PF intensity, and compressed the half-height full width of an imaged dendrite from 3.1 microns to 1.2 microns at $25-\mu \mathrm{m}$ depth. More abundant dendrites emerged at the depths of $75 \mu \mathrm{m}$ and $160 \mu \mathrm{m}$ after skull optical clearing compared with those observed through original skull (Fig. 5g-j).

Thanks to the strengthened signal level and improved image contrast after skull clearing, we were able to track the deep axons of Thy-1-GFP neurons from 250 to 700 $\mu \mathrm{m}$ (Fig. 5k and supplementary Figure 15). The vivid Thy-1-GFP neuron structures were visualized clearly after skull optical clearing, with improved imaging depth up to $>700 \mu \mathrm{m}$. In virtue of the red emission of DCBT NPs under 1300-nm three-photon excitation, multicolor fluorescence imaging was also carried out. The 3D reconstruction in Fig. 51 clearly displayed the merged image of the Thy-1-GFP neurons and DCBT NPs-labeled vessels from 0 to $600 \mu \mathrm{m}$. So far as we know, this is 
the first reported 3PF deep and simultaneous imaging of brain vessels and neurons under the intact skull.

\section{Discussion}

We proposed a solution to the high scattering effect of both skull and brain tissue through the combination of SOC technique and 3PM, and therefore realized ultra-deep cerebrovascular and neuronal imaging under intact skull. While previous skull optical clearing agents were mainly applied in two-photon fluorescence microscopy, overlooking large light absorption by water at long excitation wavelength, the VNSOCA we prepared could efficiently reduce both scattering and absorption coefficients of skull during 3PM process (Fig. 1). We also proposed a kind of AIE NPs with large three-photon absorption cross-sections in the 1300-nm excitation window (Fig. 2). Meanwhile, we confirmed its great 3PF performance on the mouse brain imaging with an open-skull glass window, achieving 1.5-mm imaging depth that was the largest one ever known, by employing the 1300-nm excitation window (Fig. 3). Such imaging depth reached hippocampal region, and was almost comparable to the depth achieved by the 3PM excited in the 1700-nm window ${ }^{31}$. In addition, 3PM on the mouse's brain with intact skull combined with SOC technique enabled 1-mm cerebrovascular imaging depth (Fig. 4), which broke the through-skull 3PM depth record using the $1700-\mathrm{nm}$ excitation window $^{34}$. Finally, the 3PF through-skull imaging depth of neuron achieved over $700 \mu \mathrm{m}$. In addition, dual-channel deep imaging of both vessels and neurons under intact skull was also realized thanks to the reduced scattering of skull and brain tissue (Fig. 5).

The combination of the two techniques is quite reasonable. On the one hand, despite that 3PM alone could reduce the skull scattering effect to a certain extent, the efficiency is not enough owing to the extremely higher scattering of skull compared with the brain tissue ${ }^{7}$. Our previously reported 3PM on the mouse brain with intact skull reached only $400-\mu \mathrm{m}^{35,36}$ depth in cerebrovascular structure imaging with excitation wavelength at $1550 \mathrm{~nm}$. On the other hand, although in vivo skull SOC could overcome the skull scattering, itself was unable to conquer the scattering effect of brain tissue. For instance, 2PM through the optical skull clearing window only reached $\sim 300-\mu \mathrm{m}$ depth in the cortex ${ }^{18}$. Therefore, the combination of SOC technique and 3PM is a rather necessary solution to through-skull ultra-deep brain imaging. Accordingly, we realized 1000- $\mu \mathrm{m}$ 3PF cerebrovascular imaging and over $700 \mu \mathrm{m}$ 3PF neuronal imaging of mouse brain with intact skull.

Even with the assistance of SOC technique and 1300-nm excited 3PM, ideal optical probes are still important for the final imaging performance. Although 3PM excited in the 1300-nm window has lower heating effect and higher excitation efficiency 
compared with that in the 1700-nm window, the stronger scattering in the 1300-nm window still constrained its imaging depth ever reported. As a solution, we proposed a new kind of AIE NPs with extraordinarily large $\sigma_{3}$ at $1300 \mathrm{~nm}$ to improve the brightness of 3PF, and eventually achieved 1.5-mm 3PM depth with open-skull glass window, which was the deepest one in the 1300-nm window.

The scattering reduction strategy presented here opens the opportunity for in vivo ultra-deep brain 3PM under the intact skull, where the imaging depth was nearly comparable with 3PM through the cranial window ${ }^{37}$. In addition, the 1300-nm excitation realized multi-color 3PM, making it possible for in vivo observation of molecular and cellular interactions in the brain under intact skull over a large depth in future. Moreover, the SOC procedure presented here is safe and easy-handling, not as difficult as craniotomy. Therefore, 3PM based on the skull optical clearing window can be widely used in various situations. We believe that in vivo 3PF imaging of hippocampus region under the intact skull of mouse brain will be realized with further reduced scattering effect of both brain tissue and skull, as well as improved 3PF probes. The combination of SOC technique and 3PM will contribute to faster and deeper imaging in scattering tissues including skull and brain.

\section{Methods}

\section{Monte Carlo simulation of NIR photon penetration in skull and brain tissue}

The Monte Carlo method was utilized to simulate the propagation of light beams in biological tissues. There are two layers of tissue in the simulation, representing the mouse skull and mouse brain tissue, respectively. We rasterized the tissue to record the scattering and absorption events of photons. In the simulation of focusing light in the tissue, photons enter the immersion media from the air, pass through the skull and focus on a specific depth in brain layer. The position of photon is randomly set within the beam radius. Meanwhile, the focal point was set at $1 \mathrm{~mm}$ depth in the skull and brain tissue after refracting in multiple tissue layers. Absorption matrix is formed to record absorbed energy of photons in tissue. The temperature profile of brain can be calculated by solving the thermal diffusion equation with absorption distribution. We set the refractive index of the skull and brain tissue as 1.369 , and the scattering anisotropy factor as 0.98 . The absorption coefficient of skull and brain tissue was set according to the water absorption coefficient in $1300 \mathrm{~nm}$ and $1700 \mathrm{~nm}$. The reduced scattering coefficient of brain tissue was calculated using the following formula ${ }^{7}$ : $\mu_{s}^{\prime}=24.2\left(\frac{\lambda}{500 \mathrm{~nm}}\right)^{-1.611}$. Thus, the brain tissue scattering coefficient in Fig. 1 was set as $5.192 \mathrm{~cm}^{-1}$ in the $1300-\mathrm{nm}$ window and $3.370 \mathrm{~cm}^{-1}$ in the $1700-\mathrm{nm}$ window respectively. The reduced scattering coefficient of skull was calculated using the following formula ${ }^{7} \mu_{s}^{\prime}=22.9\left(\frac{\lambda}{500 \mathrm{~nm}}\right)^{-0.716}$. Accordingly, the remaining skull scattering coefficient in Fig. 1 was set as $11.554 \mathrm{~cm}^{-1}(100 \%), 5.777 \mathrm{~cm}^{-1}(50 \%)$, 
$3.466 \mathrm{~cm}^{-1}(30 \%), 1.155 \mathrm{~cm}^{-1}(10 \%), 0.116 \mathrm{~cm}^{-1}(1 \%)$ in the $1300-\mathrm{nm}$ window and $9.534 \mathrm{~cm}^{-1}$ (100\%), $4.767 \mathrm{~cm}^{-1}(50 \%), 2.860 \mathrm{~cm}^{-1}(30 \%), 0.953 \mathrm{~cm}^{-1}(10 \%), 0.095$ $\mathrm{cm}^{-1}(1 \%)$ in the $1700-\mathrm{nm}$ window respectively.

\section{Preparation and vis-NIR-II absorption measurement of optical clearing agents}

The visible-NIR-II compatible skull optical clearing agents (VNSOCA) contain a saturated supernatant solution of urea and ethanol (named S1), and a high-concentration sodium dodecyl benzenesulfonate (named S2). To prepare S1, 75\% ( $\mathrm{vol} / \mathrm{vol})$ ethanol in deuteroxide solution was dropped onto the excessive urea and mixed together in a beaker. The mixture was stirred for $10 \mathrm{~min}$ and stayed for $15 \mathrm{~min}$ to dissolve urea fully. The synthesized S1 was then obtained after the supernatant solution was transferred. As to S2, the sodium dodecyl benzenesulfonate (mass = $5 \mathrm{~g}$ ) and $0.7 \mathrm{M} \mathrm{NaOH}$ in deuteroxide solution (volume $=24 \mathrm{~mL}$ ) were mixed together, under the condition of 7.2-8 pH value. Finally, both $\mathrm{S} 1$ and $\mathrm{S} 2$ solution of VNSOCA were sealed and stored at room temperature. The urea-based skull optical clearing agents (USOCA) were prepared under the similar procedure, apart from deuteroxide replaced by deionized water. The absorption spectra of both VNSOCA and USCOA were measured from 500-1880 nm with two spectrophotometers (PG2000, Ideaoptics Instruments and NIR2200-Px, Ideaoptics Instruments).

\section{Three-photon fluorescence microscopic system}

Three-photon fluorescence microscopic system included two major parts, a non-collinear optical parametric amplifier (NOPA) with wavelength-tunable femtosecond (fs) laser output and a commercial Bruker scanning microscope. The NOPA system included a $1030 \mathrm{~nm}$ fs pump laser (Spectra-Physics, Spirit) and an OPA system (Spectra-Physics, NOPA-VISIR). 1300-nm fs laser beam (115 fs, $1 \mathrm{MHz}$ ) and 1700-nm fs laser beam (203 fs, $1 \mathrm{MHz}$ ) were obtained through two amplification stages in NOPA-VISIR and introduced into the scanning microscope as excitation source. The excitation beam was focused on the sample through the objective (XLPLN25XWMP2, Olympus, NA=1.05), and the excited three-photon fluorescent signals were then collected by a GaAs PMT (H7422-40, Hamamatsu), after reflected by a $700 \mathrm{~nm}$ DMLP and passing through a $560 \mathrm{~nm}$ DMLP. The filters for red channel and green channel were FF02-641/75 (Semrock) and FF02-525/40 (Semrock).

\section{Optical Characterization of DCBT NPs}

The absorption spectra and fluorescence spectra of DCBT NPs were measured on a UV-vis scanning spectrophotometer (UV-2550, Shimadzu, Japan) and an optical fiber spectrometer (PG2000, Ideaoptics Instruments). The photoluminescence of DCBP NPs under $1300 \mathrm{~nm}$ fs excitation was carried out as following. The fs laser 
beam traveled through a focal lens $(\mathrm{f}=30 \mathrm{~mm})$ and was focused on a cuvette, which contained the dispersion of DCBT NPs. The three-photon fluorescence signal was recorded with an optical fiber spectrometer (PG2000, Ideaoptics Instruments) through an objective (XLPLN25XWMP2, Olympus, NA=1.05). The fluorescence quantum yield of DCBT NPs was measured via the comparative method with Rhodamine $6 \mathrm{G}$ in DI water as reference. Details were described in our previous work ${ }^{38}$.

\section{P-I relationship measurement and three-photon absorption cross section measurement}

The DCBT NPs were contained in the glass capillary and imaged under the three-photon fluorescence microscopic system. The 3PF images under various excitation powers were recorded. The intensity of three-photon fluorescence (I) vs the average power of $1300 \mathrm{~nm}$ fs laser $(\mathrm{P})$ was plotted to determine the P-I relationship of DCBT NPs.

To obtain the three-photon absorption cross section of DCBT NPs, the comparison method was applied. The three-photon absorption cross section of TPATCN NPs at $1550 \mathrm{~nm}^{38}$ was selected as the reference. DCBT NPs and TPATCN NPs in aqueous medium were excited by the $1550 \mathrm{~nm}$ femtosecond laser, and their three-photon fluorescence signals were collected by a photomultiplier tube (PMT). The mean three-photon fluorescence intensities were calculated by ImageJ. The $\sigma_{3}$ value of DCBT NPs was calculated by the following equation:

$$
\sigma_{31}=\sigma_{3} \frac{F_{1} \eta_{0} c_{0} n_{0}}{F_{0} \eta_{1} c_{1} n_{1}}
$$

Where $F$ is the three-photon fluorescence intensity, $\eta$ is the fluorescence quantum yield, $c$ is the molar concentration of sample, $n$ is the refractive index of the solvent, and the subscripts 1 and 0 represent DCBT NPs and TPATCN NPs, respectively.

Three-photon absorption cross section of DCBT NPs at other excitation wavelengths were referenced to the value at $1550 \mathrm{~nm}$ measured above and calculated by the following equation:

$$
\sigma_{3, \lambda}=\sigma_{3,1550} \frac{P_{1550}{ }^{3} \tau_{\lambda}^{2} F_{\lambda}}{P_{\lambda^{3} \tau_{1550} F_{1550}}}\left(\frac{\lambda}{1550}\right)^{2}
$$

Where $\sigma_{3, \lambda}$ is the wavelength dependent 3-photon absorption cross section, $\sigma_{3,1550}$ is $\sigma_{3}$ of DCBT NPs at $1550 \mathrm{~nm}, P_{1550}$ and $P_{\lambda}$ are the measured excitation powers on the sample, $\tau_{1550}$ and $\tau_{\lambda}$ are the measured pulse widths on the sample, and $F_{1550}$ and $F_{\lambda}$ are the measured three-photon fluorescence intensities with excitation at $1550 \mathrm{~nm}$ and other wavelengths, respectively.

\section{Ethical Approval}


All animal experiments performed in this study were conducted strictly in compliance with the ethical standards of the Institutional Ethical Committee of Animal Experimentation of Zhejiang University.

\section{In vivo three-photon fluorescence microscopic cerebrovascular imaging of the mouse with the cranial window}

The C57 mouse (male, 8-10 weeks old) was anesthetized by pentobarbital sodium $(0.14 \mathrm{~mL}$, mass concentration $=1 \%)$, and a cranial window with diameter of around 4 mm was produced by removing the scalp and a small piece of skull. After injected with DCBT NPs $(1.5 \mathrm{mg} / \mathrm{mL}, 200 \mu \mathrm{L})$ via the tail vein, the mouse was immobilized on a lab-built plate and imaged under the three-photon fluorescence microscopic system. The z-stack images were taken at $2-\mu \mathrm{m}$ step and the scanning speed was 2.2 $\mu \mathrm{s} /$ pixel $(512 \times 512$ pixels per frame $)$.

\section{In vivo three-photon fluorescence microscopic brain imaging of the mouse with intact skull}

Animal preparations with intact skull window and optical clearing window followed standard surgery reported previously ${ }^{39}$. The Balb/c mice (male, $\sim 6$ weeks old) with intact skull window were injected with DCBT NPs $(1.5 \mathrm{mg} / \mathrm{mL}, 200 \mu \mathrm{L})$ through tail vein. Through-skull imaging was then carried out on the three-photon fluorescence microscopic system. To evaluate the improved effects of imaging depth as well as imaging quality of 3PF microscopic cerebrovascular imaging through the established vis-NIR-II skull optical clearing window, the skull window was treated with S1 and S2 of VNSOCA to get skull optical clearing window. After that, imaging of the same field of view was conducted on the three-photon fluorescence microscopic system. Thy-1-GFP-M-line male mice ( $\sim 6$ weeks old) were treated by the aforementioned operation to obtain the skull optical clearing window. And 3PF neuronal imaging and dual-channel cerebrovascular and neuronal imaging were carried out under the same three-photon fluorescence microscopic system.

\section{Data analysis}

Image J software (Version 1.6.0, National Institutes of Health, USA) was applied for quantitative analysis of each fluorescent image. Origin Pro software (Version 9.0, OriginLab Company, USA), Imaris (Version 9.0, Oxford Instruments) and Adobe Illustrator CC (Version 2018) was applied for graph generation.

\section{References}

1. Zhang, Z.Q. et al. Altered functional-structural coupling of large-scale brain networks in idiopathic generalized epilepsy. BRAIN 134, 2912-2928 (2011). 
2. Stern, Y. et al. Whitepaper: Defining and investigating cognitive reserve, brain reserve, and brain maintenance. ALZHEIMERS \& DEMENTIA 16, 1305-1311 (2020).

3. Alexander-Bloch, A., Giedd, J.N. \& Bullmore, E.T. Imaging structural co-variance between human brain regions. NATURE REVIEWS NEUROSCIENCE 14, 322-336 (2013).

4. Reiman, E.M. et al. Brain imaging and fluid biomarker analysis in young adults at genetic risk for autosomal dominant Alzheimer's disease in the presenilin 1 E280A kindred: a case-control study. LANCET NEUROLOGY 11, 1048-1056 (2012).

5. Horn, A. et al. Connectivity Predicts Deep Brain Stimulation Outcome in Parkinson Disease. ANNALS OF NEUROLOGY 82, 67-78 (2017).

6. Orru, G., Pettersson-Yeo, W., Marquand, A.F., Sartori, G. \& Mechelli, A. Using Support Vector Machine to identify imaging biomarkers of neurological and psychiatric disease: A critical review. NEUROSCIENCE AND BIOBEHAVIORAL REVIEWS 36, 1140-1152 (2012).

7. Jacques, S.L. Corrigendum: Optical properties of biological tissues: a review. Physics in Medicine and Biology 58, 5007-5008 (2013).

8. Xia, J., Yao, J.J. \& Wang, L.V. Photoacoustic Tomography: Principles and Advances. PROGRESS IN ELECTROMAGNETICS RESEARCH-PIER 147, 1-22 (2014).

9. Attia, A.B.E. et al. A review of clinical photoacoustic imaging: Current and future trends. PHOTOACOUSTICS 16 (2019).

10. Nie, L.M. \& Chen, X.Y. Structural and functional photoacoustic molecular tomography aided by emerging contrast agents. CHEMICAL SOCIETY REVIEWS 43, 7132-7170 (2014).

11. Shang, Z.M., Ding, Z.H., Wang, L. \& Liu, Y. Path length coded coherence combination for three-dimensional superresolution. ACTA PHYSICA SINICA 60 (2011).

12. Han, T. et al. Optical Coherence Microscopy and Its Application. CHINESE JOURNAL OF LASERS-ZHONGGUO JIGUANG 47 (2020).

13. Chen, P. et al. Fingerprint Anti-counterfeiting Method Based on Optical Coherence Tomography and Optical Micro-angiography. Acta Photonica Sinica 48 (2019).

14. Han, T. et al. Optical Coherence Microscopy and Its Application. Chinese Journal of Lasers 47 (2020).

15. Holtmaat, A. et al. Long-term, high-resolution imaging in the mouse neocortex through a chronic cranial window. Nat Protoc 4, 1128-1144 (2009).

16. Yang, G., Pan, F., Parkhurst, C.N., Grutzendler, J. \& Gan, W.B. Thinned-skull cranial window technique for long-term imaging of the cortex in live mice. Nat Protoc 5, 201-208 (2010).

17. Yu, X. \& Zuo, Y. Two-photon in vivo imaging of dendritic spines in the mouse cortex using a thinned-skull preparation. J Vis Exp, 51520 (2014).

18. Zhang, C. et al. A large, switchable optical clearing skull window for 
cerebrovascular imaging. Theranostics 8, 2696-2708 (2018).

19. Zhao, Y.J. et al. Skull optical clearing window for in vivo imaging of the mouse cortex at synaptic resolution. Light Sci Appl 7, 17153 (2018).

20. Xu, H.-T., Pan, F., Yang, G. \& Gan, W.-B. Choice of cranial window type for in vivo imaging affects dendritic spine turnover in the cortex. Nature Neuroscience 10, 549-551 (2007).

21. Wang, T. \& Xu, C. Three-photon neuronal imaging in deep mouse brain. Optica 7 (2020).

22. Horton, N.G. et al. In vivo three-photon microscopy of subcortical structures within an intact mouse brain. Nature Photonics 7, 205-209 (2013).

23. König, K. et al. in Multiphoton Microscopy in the Biomedical Sciences XVIII (2018).

24. Ouzounov, D.G. et al. In vivo three-photon imaging of activity of GCaMP6-labeled neurons deep in intact mouse brain. Nature Methods 14, 388-390 (2017).

25. Wang, T. et al. Three-photon imaging of mouse brain structure and function through the intact skull. Nature Methods 15, 789-792 (2018).

26. Hontani, Y., Xia, F. \& Xu, C. Multicolor three-photon fluorescence imaging with single-wavelength excitation deep in mouse brain. Science Advances 7, eabf3531.

27. Wang, Y. et al. Measurement of absorption spectrum of deuterium oxide (D2O) and its application to signal enhancement in multiphoton microscopy at the 1700-nm window. Applied Physics Letters 108, 021112 (2016).

28. Cheng, L.C., Horton, N.G., Wang, K., Chen, S.J. \& Xu, C. Measurements of multiphoton action cross sections for multiphoton microscopy.

29. Li, D. et al. AIE-nanoparticle assisted ultra-deep three-photon microscopy in the in vivo mouse brain under $1300 \mathrm{~nm}$ excitation. Materials Chemistry Frontiers 5, 3201-3208 (2021).

30. Liu, H. et al. In Vivo Deep-Brain Structural and Hemodynamic Multiphoton Microscopy Enabled by Quantum Dots. Nano letters (2019).

31. Liu, M. et al. Binary Organic Nanoparticles with Bright Aggregation-Induced Emission for Three-Photon Brain Vascular Imaging. Chemistry of Materials 32, 6437-6443 (2020).

32. Streich, L. et al. High-resolution structural and functional deep brain imaging using adaptive optics three-photon microscopy. Nat Methods (2021).

33. Xia, F. et al. Short-Wave Infrared Confocal Fluorescence Imaging of Deep Mouse Brain with a Superconducting Nanowire Single-Photon Detector. ACS Photonics (2021).

34. Chen, Y. et al. Coherent Raman Scattering Unravelling Mechanisms Underlying Skull Optical Clearing for Through-Skull Brain Imaging. Analytical Chemistry 91, 9371-9375 (2019).

35. Alifu, N. et al. NIR-IIb excitable bright polymer dots with deep-red emission for in vivo through-skull three-photon fluorescence bioimaging. Nano Research 13, 2632-2640 (2020). 
36. Wang, Y. et al. Aggregation-Induced Emission Luminogen with Deep-Red Emission for Through-Skull Three-Photon Fluorescence Imaging of Mouse. ACS Nano 11, 10452-10461 (2017).

37. Wang, S. et al. In Vivo Three-Photon Imaging of Lipids using Ultrabright Fluorogens with Aggregation-Induced Emission. Adv Mater, e2007490 (2021).

38. Wang, Y. et al. Bright AIE Nanoparticles with F127 Encapsulation for Deep-Tissue Three-Photon Intravital Brain Angiography. Advanced healthcare materials 6 (2017).

39. Li, D.Y. et al. Visible- near infrared -II skull optical clearing window for in vivo cortical vasculature imaging and targeted manipulation. Journal of Biophotonics 13 (2020).

\section{Acknowledgements}

This work was supported by National Natural Science Foundation of China (61975172, 82001874, and 21974104) and Natural Science Foundation of Zhejiang Province (LR17F050001).

The authors thank Dandan Song in the Center of Cryo-Electron Microscopy (CCEM), Zhejiang University for her technical assistance on STEM.

\section{Author contributions}

J.Q. and M.H. conceived the idea and designed the experiments. D.L. and D.Z. provided skull optical clearing technique guidance. Z.Z. provided the fluorescence probes. H.Z. and W.X. provided help on 3PM setup system. T.W performed the simulation. L.Z. provided guidance on cranial surgery. Z.H. helped to perform experiment in Fig. 5. M.H., D.L., W.G. Z.H. Z.F. performed the experiments. M.H., D.L., W.G., S.P. and J.Q. analyzed the data and wrote the manuscript. All authors discussed the results and commented on the manuscript.

\section{Competing interests}

The authors declare no competing interests.

\section{Keywords}

Deep brain imaging, scattering reduction, through skull, optical clearing, three-photon microscopy, aggregation-induced emission 

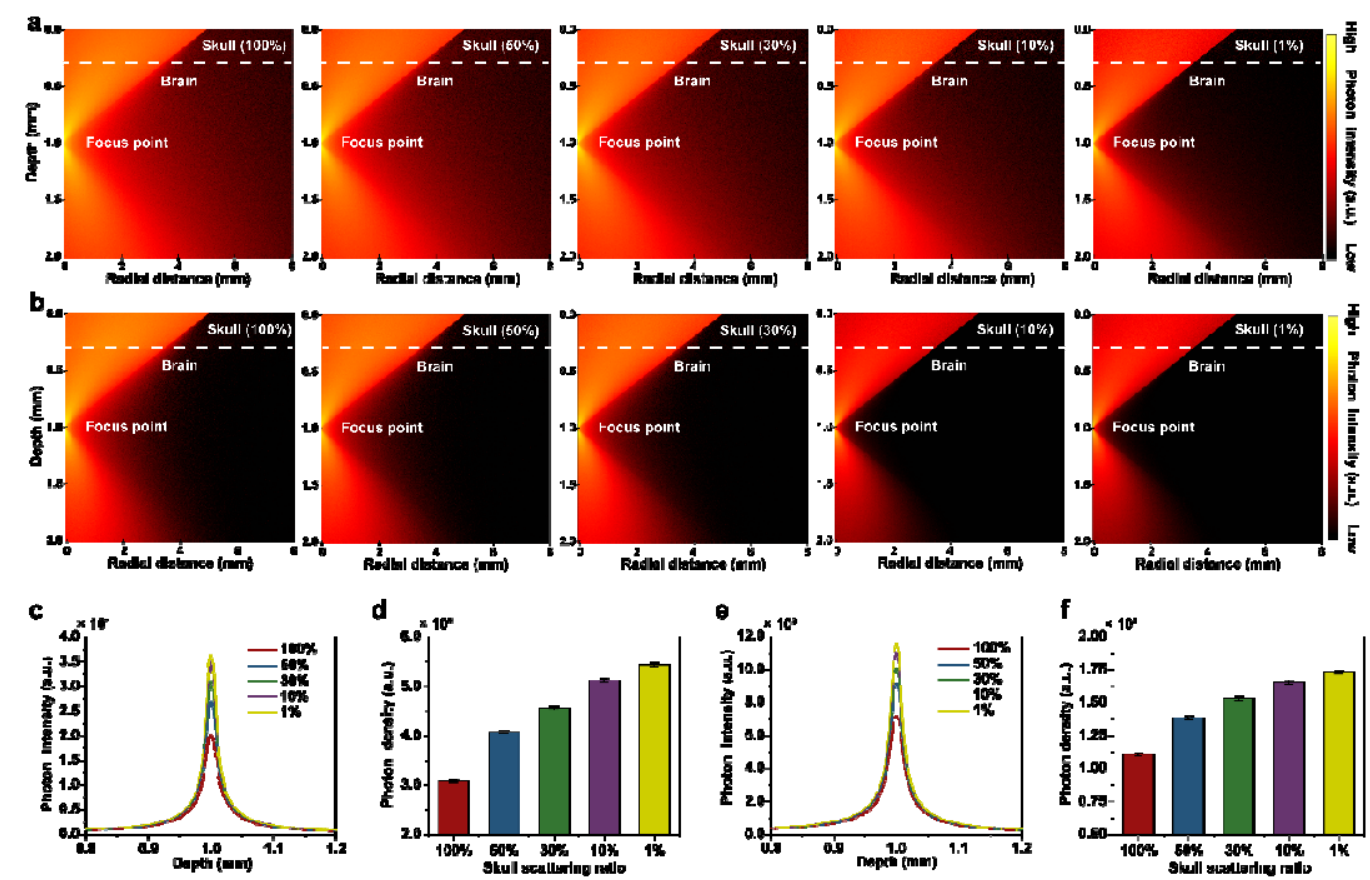

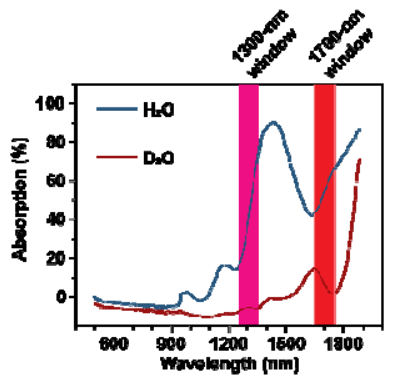

d

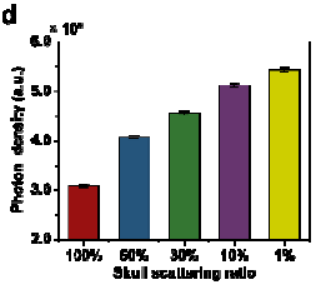

- $120^{\times 100}$ f

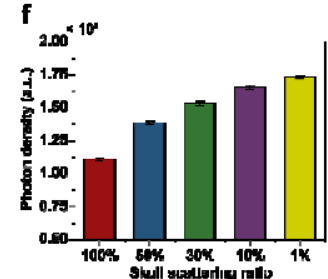

$\mathbf{h}$

I
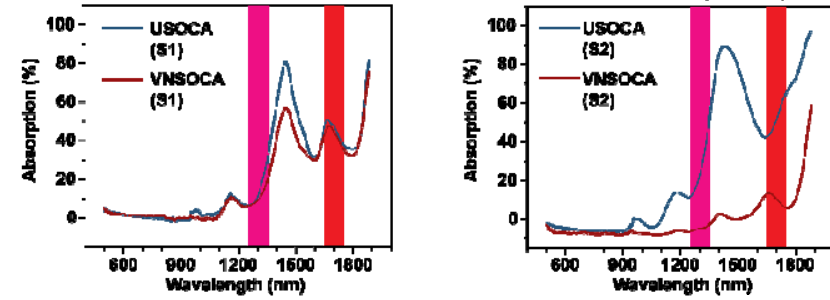

Fig. 1 Skull scattering reduction improved performance of through-skull 3PM. a, b R-Z plane photon distribution simulation of excitation light in the 1300-nm window (a) and the 1700-nm window (b) penetrating through skull and brain tissue with different remaining skull scattering ratio $(100 \%$ to $1 \%)$. Focal depth $=1 \mathrm{~mm}$. c, e Photon intensity (number of photons at focal plane) profiles along the depth (Radial distance $=0 \mathrm{~mm}$ ) from $0.8 \mathrm{~mm}$ to $1.2 \mathrm{~mm}$ with different remaining skull scattering ratios (100\% to $1 \%$ ) in the $1300-\mathrm{nm}$ window (c) and the $1700-\mathrm{nm}$ window (e). d, f Photon density (number of photons divided by focal spot area at focal plane) at the focal point with different remaining skull scattering ratio (100\% to $1 \%$ ) in the 1300-nm window (d) and the 1700-nm window (f). g Absorption spectra of $\mathrm{H}_{2} \mathrm{O}$ and deuterium oxide $\left(\mathrm{D}_{2} \mathrm{O}\right)$. h, i Absorption spectra of solution 1 (S1) and immersed solution 2 (S2) of urea-based skull optical clearing agents (USOCA) and visible-NIR-II compatible skull optical clearing agents (VNSOCA). (Optical length = $1 \mathrm{~mm})$. 


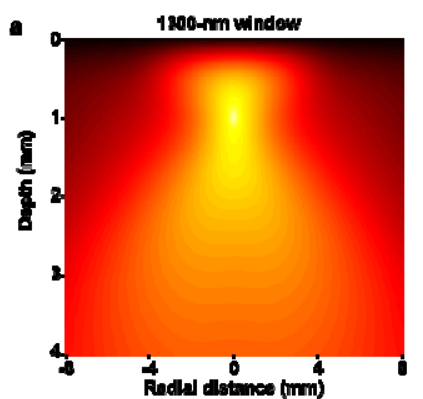

c

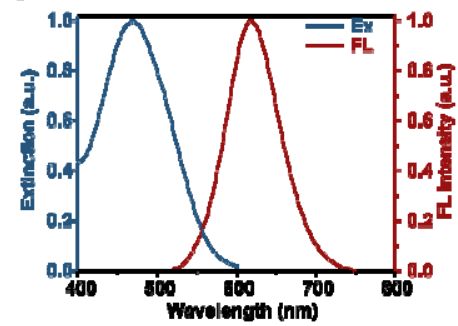

1

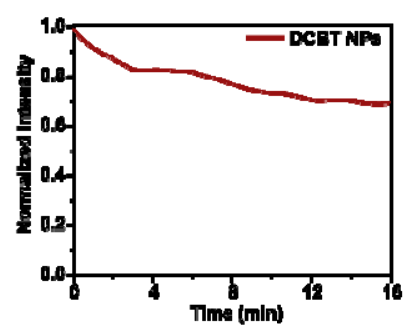

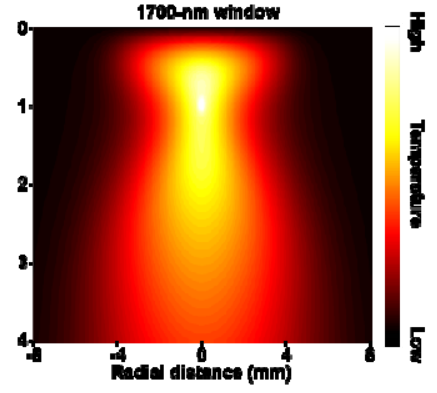

d

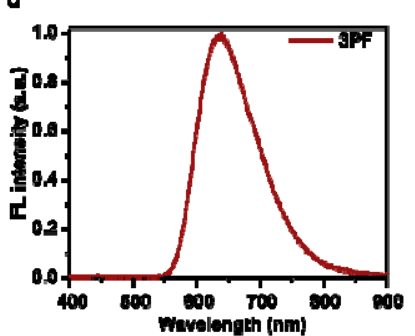

g

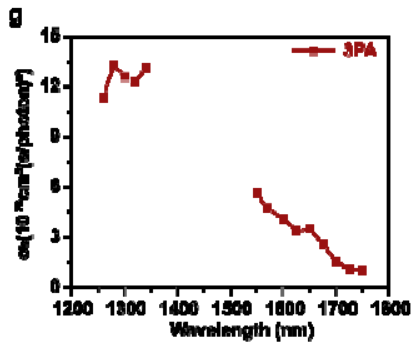

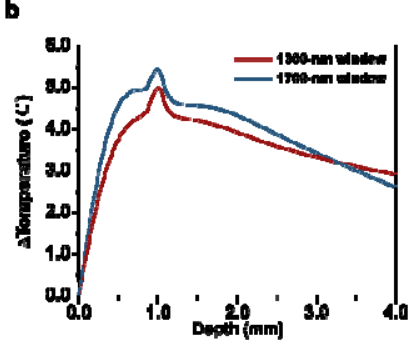
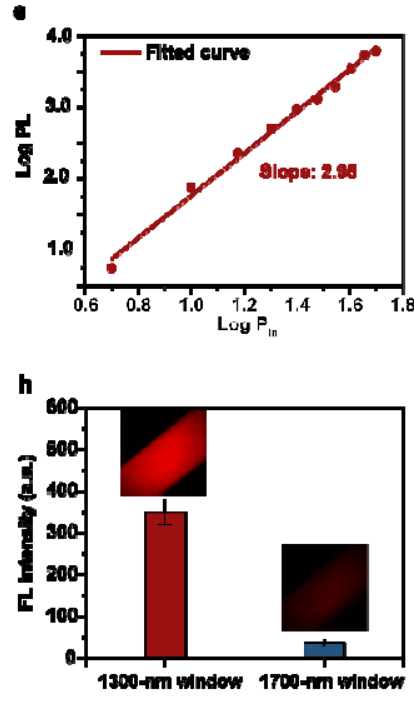

Fig. 2 The advantages of the 1300-nm excitation window over 1700-nm excitation

window. a Monte Carlo simulation of temperature rising distribution caused by tissue absorption in the 1300-nm window and 1700-nm window. (Focal depth $=1 \mathrm{~mm}$ ). b Temperature rising distribution line versus depth at the position of $0 \mathrm{~mm}$ radial distance. c Normalized extinction and PL spectra of DCBT NPs. d 3PF spectrum of DCBT NPs under the excitation of $1300 \mathrm{~nm}$ fs laser. e Power dependence of fluorescence intensity of DCBT NPs under the $1300 \mathrm{~nm}$ fs excitation intensity. $\mathbf{f}$ Photostability of DCBT NPs under the continuous irradiation of $1300 \mathrm{~nm}$ fs laser (Average power: $7 \mathrm{~mW}$ ). $\mathrm{g}$ Measured three-photon absorption cross-sections of DCBT NPs from $1260 \mathrm{~nm}$ to $1750 \mathrm{~nm}$. h 3PF intensity of DCBT NPs under the excitation of 1300-nm window and 1700-nm window. Insert: 3PF images of DCBT NPs filled in the glass capillary. 

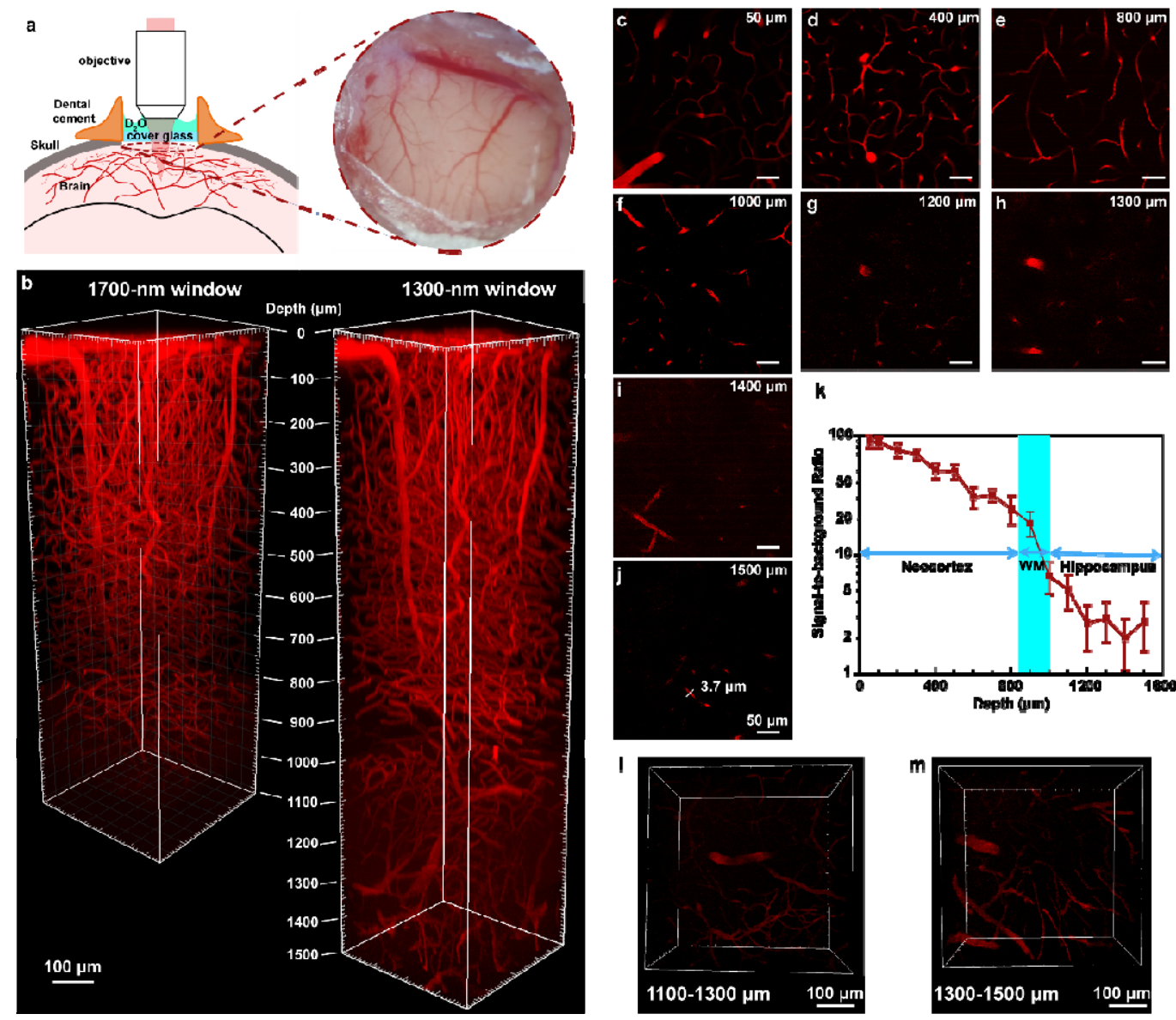

$\mathbf{k}$
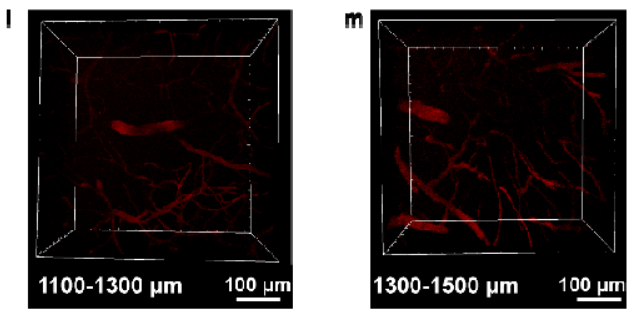

Fig. 3 In vivo deep 3PF brain vasculature imaging of the mouse with the open-skull glass window. a Schematic of mouse head imaging with the open-skull window. The bright field picture of mouse brain was shown at right position. b 3D reconstruction of the cerebrovascular imaging under 3PM excited using the 1700-nm window $(0-1100 \mu \mathrm{m})$ and 1300-nm window $(0-1500 \mu \mathrm{m})$. c-j 3PF imaging of the mouse brain vasculature at various depths excited in the $1300-\mathrm{nm}$ window $\mathbf{k}$ The Signal-to-background ratio (SBR) as a function of imaging depth. The white matter (WM) region of the mouse brain, where the SBR decreased sharply, is indicated in blue. I-m 3D reconstruction of the cerebrovascular imaging in 1100-1300 $\mu \mathrm{m}$ and $1300-1500 \mu \mathrm{m}$ depth. 

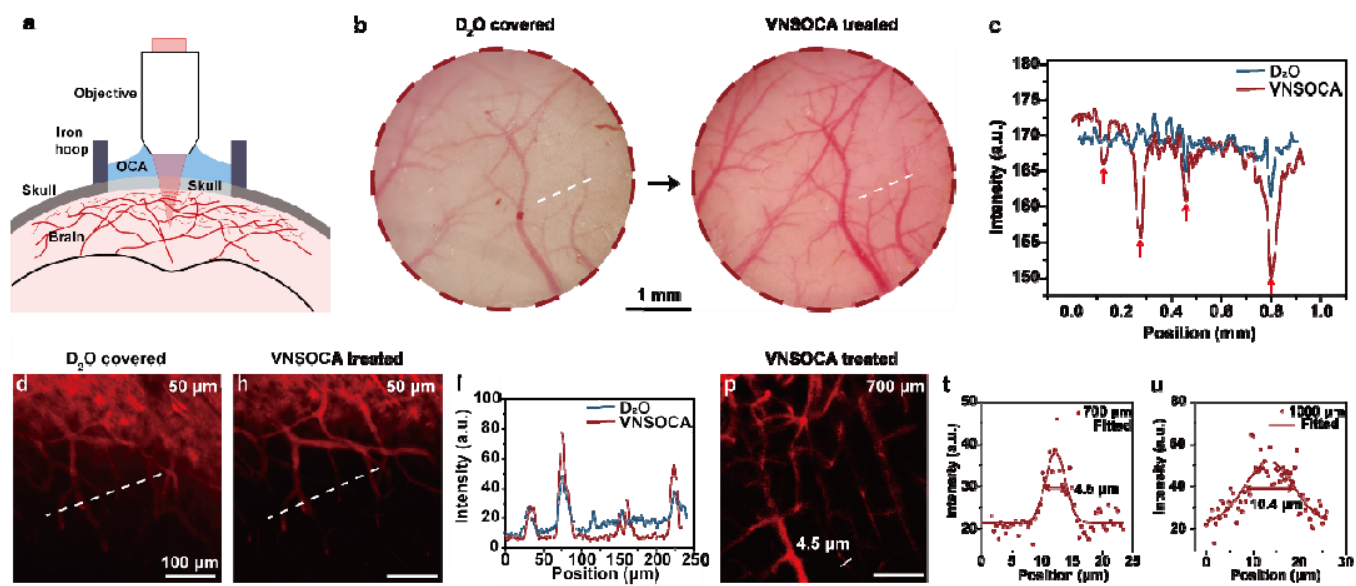

VNSOCGA treoted

VNSOCA trented
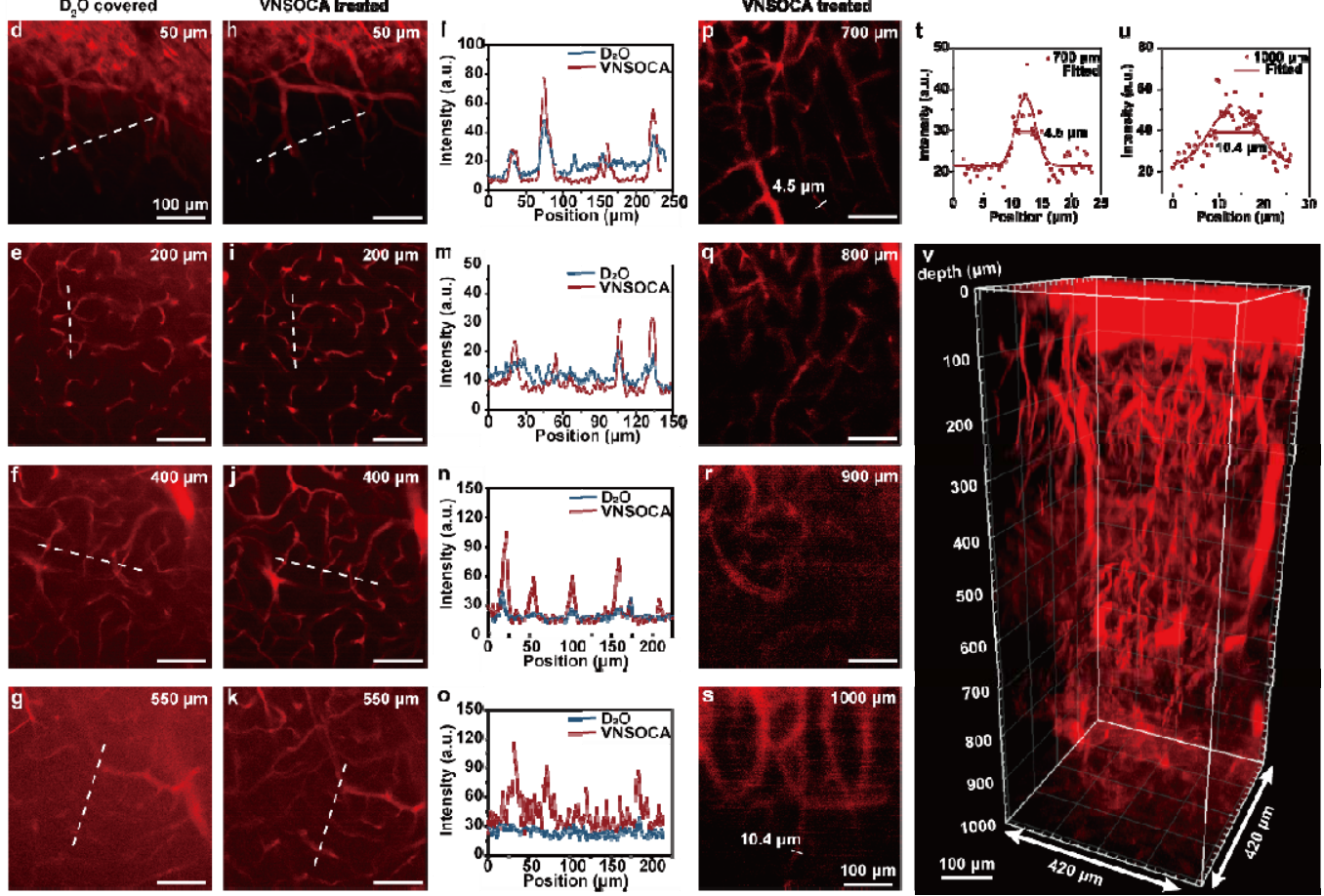

Fig. 4 In vivo deep 3PF brain vasculature imaging of the mouse with the optical clearing window. a Schematic of mouse head imaging with the optical clearing window. b Typical bright field pictures of the mouse brain vasculature before and after skull clearing. c Intensity profiles along the white dashed lines across the vasculature in (b). The arrows indicated the positions of vessels. d-g 3PF imaging of the mouse brain vasculature at various depths with original skull. h-k 3PF imaging of the mouse brain vasculature at various depths after skull clearing. l-o Intensity profiles along the white dashed lines across the brain vasculature in (d)-(k) respectively. p-s Deep 3PF images of the mouse brain vasculature after skull clearing. t-u FWHM analysis of the brain vasculature at the depths of $700 \mu \mathrm{m}(\mathrm{t})$ and $1000 \mu \mathrm{m}$ (u). $\mathbf{v} 3 \mathrm{D}$ reconstruction of the cerebrovascular imaging with the optical clearing window $(0-1000 \mu \mathrm{m})$. 

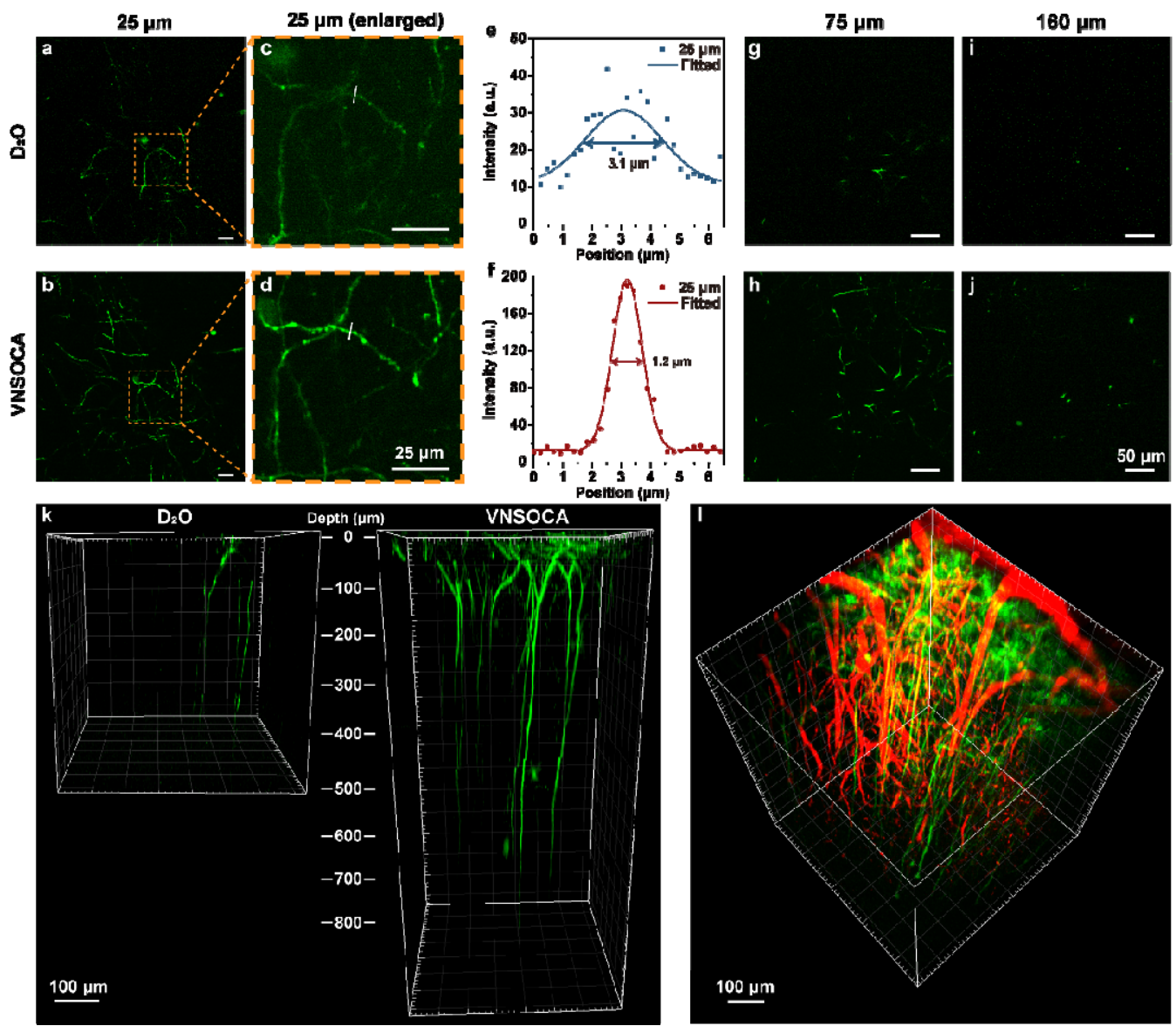

Fig. 5 In vivo deep 3PF brain neuron imaging and neuron-vessels dual channel imaging of the mouse with the optical clearing window. a-b 3PF images of the mouse brain neurons at $25-\mu \mathrm{m}$ depth before and after skull clearing. c-d Enlarged 3PF images of the mouse brain neurons in (a) and (b). e-f Intensity and FWHM analysis of the brain neurons in (c) and (d). $\mathbf{g}-\mathbf{j} 3 \mathrm{PF}$ images of the mouse brain neurons before and after skull clearing at depth of $75 \mu \mathrm{m}$ and $160 \mu \mathrm{m}$. $\mathbf{k}$ 3D reconstruction of the 3PF neuronal imaging through the original skull $(0-400 \mu \mathrm{m})$ and the skull optical clearing window $(0-800 \mu \mathrm{m})$. 1 3D reconstruction of the neuron-vessels dual channel imaging of the mouse with the optical clearing window $(0-600 \mu \mathrm{m})$. 